\title{
Aging Characteristics of Lithium Titanate Batteries under Ultra- high Discharge Rate
}

\author{
Zehui Liu ${ }^{1,2}$, Chu Wang ${ }^{1,2}$, Chen Miao ${ }^{1,2}$, Yinghui Gao ${ }^{1}$ and Yaohong Sun ${ }^{1,2^{*}}$ \\ ${ }^{1}$ Institute of Electrical Engineering, Chinese Academy of Sciences, Beijing, 100190, China \\ ${ }^{2}$ University of Chinese Academy of Sciences, Beijing, 100049, China
}

\begin{abstract}
The aging characteristics of lithium titanate batteries under ultra-high discharge rate were studied in this paper. The discharge characteristics is highly temperature-dependent under 55C discharge rate. The capacity retention rate is about $73 \%$ after 100 cycles of $55 \mathrm{C}$ discharge. The in-situ analysis incremental capacity analysis (ICA) was used to analyze the aging mechanism, and it was found that the main reason for the capacity fading was the loss of active material of the positive electrode, which leads to an increase of polarization resistance under $55 \mathrm{C}$ discharge rate.
\end{abstract}

\section{Introduction}

Lithium-ion battery is an environment-friendly energy form with high specific energy, specific power and long cycle life, which is widely used in rail transit, aerospace and military industries[1,2]. Lithium-ion batteries are divided into energy type and power type. The energy type uses ternary materials or lithium iron phosphate as the positive electrode and graphite as the negative $\mathrm{s}$ ae electrode, while lithium titanate or niobium titanium material are used as negative electrode in power type lithium-ion batteries, which can realize rapid charge and discharge. Power type lithium-ion batteries have great advantages in applications that requiring large current and high power output.

Compared with energy lithium-ion batteries, lithium titanate battery has a wider temperature range, longer cycle life and higher safety[3,4]. Generally, the capacity degradation of lithium-ion batteries is caused by the loss of anode and cathode materials and the loss of recyclable lithium[5,6]. For energy type batteries, the aging mechanism is often attributed to the loss of anode material. However, the anode materials of lithium titanate batteries have high structural stability, and the aging mechanism of this kind of battery is mainly independent of the anode material. Liu et al.[7] mentioned that the capacity decline of lithium-titanate batteries at room temperature is mainly caused by the loss of cathode materials, and the life decay rate of lithium-titanate batteries is slower under high discharge rate due to the low capacity throughput. Svens et al.[8] found that the capacity loss of lithium titanate batteries at $55^{\circ} \mathrm{C}$ was mainly due to the dissolution of $\mathrm{Mn}^{2+}$ in the cathode material and the structural change of lithium cobaltate by using the incremental capacity analysis (ICA), differential voltage analysis (DVA) and combined with disassembling method. However, the current research on the aging mechanism of lithium titanate batteries is still not sufficient enough, especially for the high charging and discharging rate conditions.

In this paper, the commercial lithium titanate batteries were subjected to a cyclic aging test at $55 \mathrm{C}$ discharge rate. The parameters of cyclic aging process such as voltage and current curve, ohmic internal resistance, discharge capacity and discharge temperature rise were studied, and the aging mechanism was explored by ICA method.

\section{Experimental}

A commercial 15 Ah lithium titanate battery was used in this article, and detailed parameters are shown in table 1 . The whole test system is shown in figure 1. The discharge is carried on Chroma 17212 programmable charge and discharge tester, the applied voltage range is $0 \sim 5 \mathrm{~V}$, the current response is within $10 \mu \mathrm{s}$, and it can realize 1200 A discharge via channel parallel output. And a T-type thermocouple was mounted on the core of the battery to measure the temperature during the discharge.

The aging test of LTO battery contains ten times of the process of three times of $1 \mathrm{C}$ discharge tests and ten times of $55 \mathrm{C}$ discharge tests. And the test procedure is shown as following: 1) CCCV charge: the battery was charged at $1 \mathrm{C}$ constant current charge until the cut-off voltage was reached, and then a constant voltage charged was used until the current dropped to 0.05C. 2) Stand for 1h. 3) Discharge at a pre-set discharge rate until the discharge cut-off voltage. 4)Stand for 1h. 5) Repeat the working steps above.

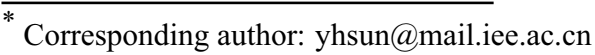


Table 1. Technical parameters of the LTO battery.

\begin{tabular}{llc}
\hline & SPECIFICATIONS & VALUES \\
\cline { 2 - 3 } & Rated Capacity & $15 \mathrm{Ah}$ \\
& Rated Voltage & $2.35 \mathrm{~V}$ \\
& Operating Voltage & $1.50 \mathrm{~V} \sim 2.75 \mathrm{~V}$ \\
& Size & $216 \mathrm{~mm} \times 127 \mathrm{~mm} \times 6.1 \mathrm{~mm}$ \\
& Operating Temperature & $-20^{\circ} \mathrm{C} \sim 60^{\circ} \mathrm{C}$ \\
& Positive Electrode & $\mathrm{NCM}$ \\
& Negative Electrode & Lithium titanate oxide \\
\hline
\end{tabular}

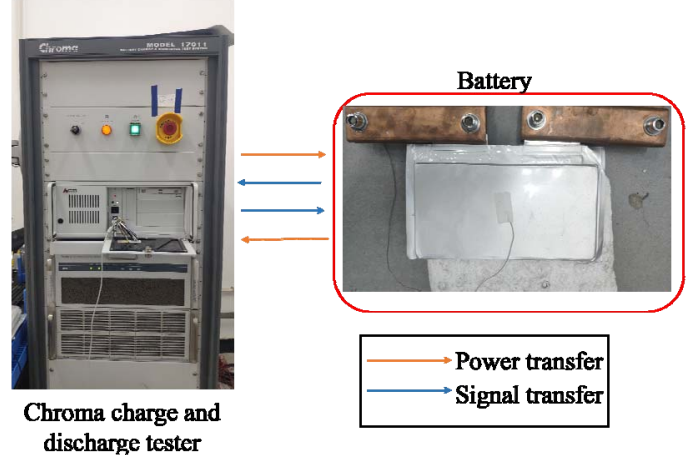

Figure 1. A schematic experimental diagram.

\section{Results and Discussion}

\subsection{Discharge characterisctics}

The discharge characteristics of the LTO battery are shown in figure 2 . It discharges from $1 \mathrm{C}$ to $70 \mathrm{C}$ at $20{ }^{\circ} \mathrm{C}$. The polarization rate is increasing with the increase of discharge rates[9], therefore, it will be sooner to reach the cut-off voltage under high discharge rates. The battery sustains a high discharge capacity over $90 \%$ when the discharge rates are under $30 \mathrm{C}$. The discharge capacity decreases sharply when the discharge rates over $30 \mathrm{C}$, which is caused by the high polarization rates. The discharge curve of $55 \mathrm{C}$ has a significant voltage platform compared with that of over $55 \mathrm{C}$, therefore, it is chosen to analyze the aging mechanism. The discharge capacity of $55 \mathrm{C}$ at $20^{\circ} \mathrm{C}$ is $5.70 \mathrm{Ah}$, and the discharge time is $24.9 \mathrm{~s}$.

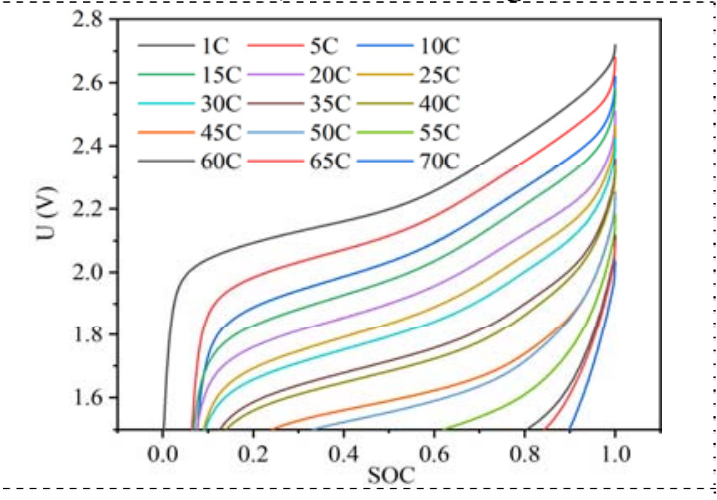

Figure 2. The discharge characteristics under different discharge rates.

\subsection{Aging Characteristics}

The evolution of discharge curve under aging test is shown in figure 3. The whole tendency is that the discharge curve moves downward in the aging test and the discharge time is decreasing, which means the internal resistance is increasing. The curve cycle 30 has a large internal resistance, which is affected by the low temperature. The internal resistance contains ohmic resistance and polarization resistance. The ohmic resistance is decided by the contact resistance between the materials. The polarization resistance is decided by the charge transfer impedance and ion diffusion impedance during the charge and discharge process, which is related to the charge and discharge rate.

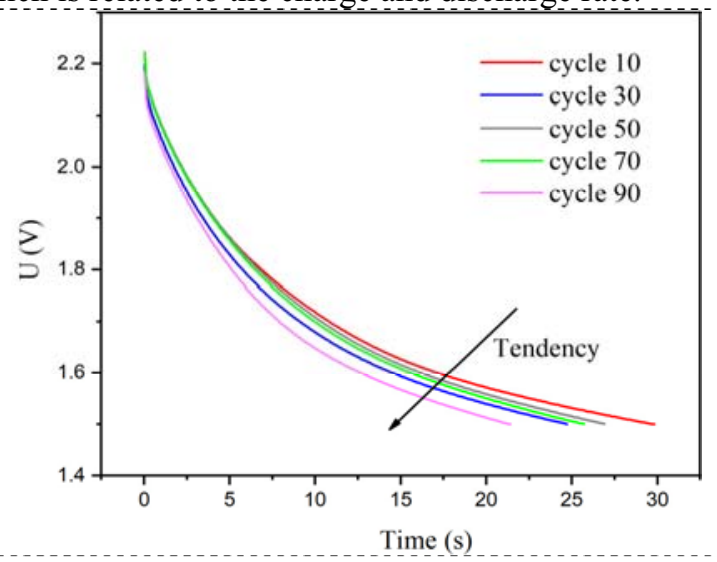

Figure 3. The evolution of discharge curve under aging test.

The evolution of discharge capacity under aging test is shown in figure 4 . The temperature of the battery before the discharge process is given as well. And each "star" denotes the location of ten times of 55C discharge. The capacity during the aging test is in a decreasing tendency, when the battery is cycled for 100 times, the discharge capacity sustains about $73 \%$. Of which, the capacity of 20-30 cycle discharge and 70-90 cycle discharge drastically below the tendency, which is caused by the fluctuation of temperature. The capacity of $1 \mathrm{C}$ discharge changes within a small range (except for 9th discharge, affected by the low temperature), which means few loss of lithium inventory (LLI) exist. The 
polarization process under high discharge rate is strongly affected by the temperature. The temperature is over $20{ }^{\circ} \mathrm{C}$, the conductivity of electrode material is higher, the electrochemical characteristics is better, and the discharge capacity released is higher.

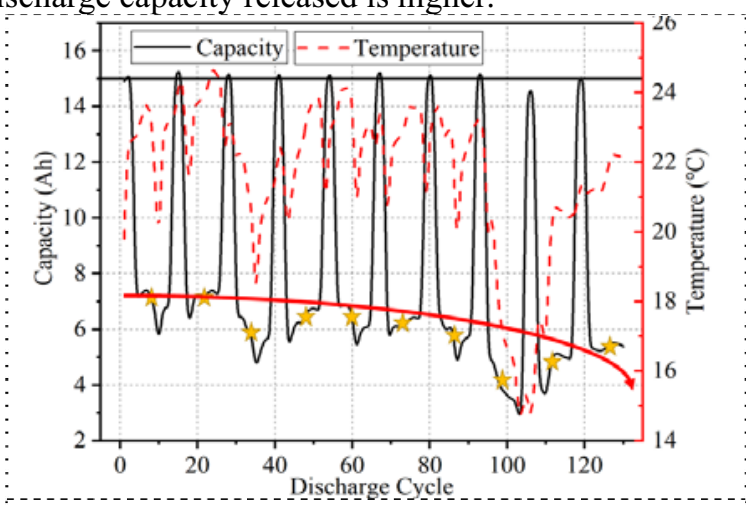

Figure 4. The evolution of discharge capacity under aging test.

The evolution of temperature rise and ohmic resistance under $55 \mathrm{C}$ test is shown in figure 5 . The variation of ohmic resistance is highly temperaturedependent in this paper, and it could not figure out the variation is whether increasing or decreasing during the aging test under the fluctuation of ohmic resistance. The fluctuation tendency of initial temperature, final temperature and temperature rise are the same, while the fluctuation tendency of ohmic resistance is the opposite. Two significant maximum appear during cycle 20-40 and cycle 70-90 at the low temperature region, which correspond to the two minimum low temperature rise region.

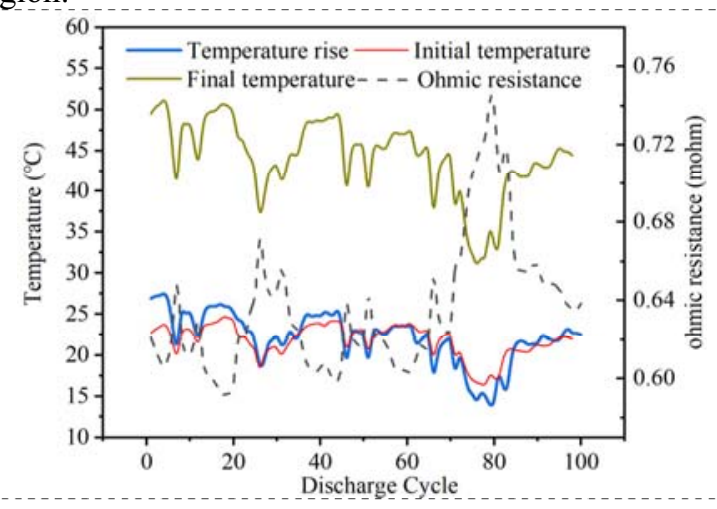

Figure 5. The evolution of temperature rise and ohmic resistance under $55 \mathrm{C}$ test.

When the temperature is lower, the conductivity of electrode material is lower, therefore, the ohmic resistance is higher, and the polarization rate is higher. Under high discharge rates, the increase of internal resistance will lead to a shorter time to reach the cut-off voltage, which also means a lower discharge capacity. The percentage of heat generation in the whole discharge energy will increase with a high internal resistance[10], however, the discharge energy is decreased. In this study, the decrease rate of discharge energy is higher than the increase rate of heat generation, therefore, the decrease of temperature rise is obtained.
Long-term cycle lithium-ion batteries will inevitably lead to internal material damage, structural decay, particle stripping and even electrode delamination. ICA is a technology that can realize in situ analysis of battery decay mechanism in the whole life cycle by giving $\mathrm{d} Q / \mathrm{d} U-U$ curves. In the cycle process, different decline mechanisms will leave corresponding marks on the ICA characteristic curve.

The analysis of capacity increment curve is shown in figure 6. Generally, more accurate analysis results can be obtained by using smaller current. However, due to the self-repairing effect of electrode material and longer experimental time in the process of small current charging and discharging, the state analysis of the battery under high rate cycle is affected, so $1 \mathrm{C}$ rate is selected for ICA. Due to the high coincidence of IC curves, in order to better display the changes of characteristic curves in the process, the IC curves were divided into two parts for display, and the position of characteristic peaks was enlarged.

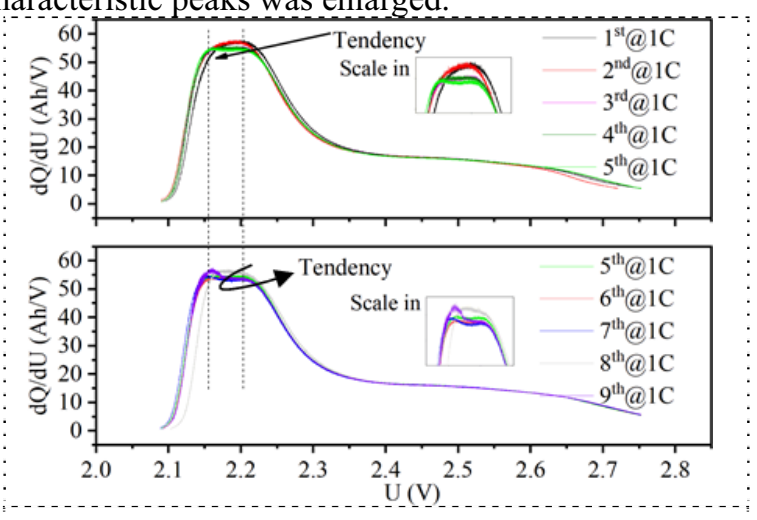

Figure 6. ICA characteristic curve during the aging test.

Each 1C discharge curve corresponds to the state before 10 times of $50 \mathrm{C}$ cycles. According to the ICA curve from 1 st to 7 th, it can be seen that in the first 60 cycles, the ICA curve moved left and down. The 8th curve proceeded under the condition of too low temperature, so the trend was different from other curves. Compared with the 1st-7th curve, the 9th curve moved right and up, however, it still moved left and down compared with the 8 th curve. The change of the peak position of the curve means the crystal structure change of the electrode material, the change of the intensity of the curve peak represents the change of the active material, and the movement of the horizontal axis corresponding to the curve means the change of the internal resistance of the battery. A shift to the left represents a decrease in internal resistance. However, ICA carries out under $1 \mathrm{C}$ charge rate and the polarization rate is small compared to high discharge rate. The internal resistance is mainly decided by the ohmic resistance. Therefore, in the aging cycle, in order to meet the demand of high rate discharge, the ohmic resistance of the battery decreases, at the same time the cathode active material of lithium titanate battery falls off and the active material reduced, which leads to an increase of polarization resistance under high discharge rate. However, due to the abnormal reduction of discharge 
capacity in the 8th discharge process, the positive electrode material self-recovery effect appeared. After that, the ICA standard was re-established. Compared with the eighth ICA active material, the internal resistance was reduced. However, this anomaly does not affect the decreasing trend of discharge capacity during the whole aging process. In conclusion, during the discharge cycle, the battery is in an activated state, and the corresponding internal resistance decreases. However, the loss of cathode materials leads to an increase of the polarization resistance under high discharge rate, which leads to the capacity fading.

\section{Conclusion}

A commercial lithium titanate battery with 55C discharge rate cycle was studied in this paper. The discharge capacity and discharge temperature rise of the battery decrease with the cycling process. The discharge curve and the ohmic resistance of the battery are very temperature sensitive, the lower the temperature is, the higher the voltage drop of the discharge curve is, and the greater the ohmic resistance is. ICA analysis shows that the height of the characteristic peak decreases and the position of the characteristic peak moves to the left during the aging process of the battery, indicating that the loss of cathode material and the decrease of ohmic internal resistance. And the main reason of aging is the loss of cathode material, which leads to an increase of polarization resistance under high discharge rate. The decrease of ohmic resistance reduction is more favorable to high rate discharge, but the increase of polarization rate is not. Due to the influence of low temperature, an incomplete discharge occurs during the cycle, and the electrode material appears self-recovery phenomenon, but this phenomenon does not reverse the reduction of high rate discharge capacity.

\section{Acknowledgement}

Thanks are due to M. Chen for modification of thesis writing, C. Wang and Y.H. Gao for assistance with the experiment, Y.H. Sun for the paper review and the financial support.

\section{References}

1. S. Chacko, J.M. Chung, Thermal modeling of Li-ion polymer battery for electric vehicle drive cycles, J. Power Sources 213, 296-303 (2012)

2. K. Shah, V. Vishwakarma, A. Jain, Measurement of Multiscale Thermal Transport Phenomena in Li-ion Cells: A Review, J. Electrochem. Energy 13, 030801 (2016)

3. G. Xu, P. Han, S. Dong, et al. $\mathrm{Li}_{4} \mathrm{Ti}_{5} \mathrm{O}_{12}$-based energy conversion and storage systems: Status and prospects, Coordin. Chem. Rev. 343, 139-184 (2017)

4. P. Schröer, E. Khoshbakht, T. Nemeth, et al, Adaptive modeling in the frequency and time domain of high-power lithium titanate oxide cells in battery management systems, J. Energy Storage, 32, 101966 (2020)

5. H. Zheng, Q. Sun, G. Liu, et al, Correlation between dissolution behavior and electrochemical cycling performance for $\mathrm{LiNi}_{1 / 3} \mathrm{Co}_{1 / 3} \mathrm{Mn}_{1 / 3} \mathrm{O}_{2}$-based cells, $\mathrm{J}$. Power Sources, 207, 134-140 (2012)

6. Y.B. He, F. Ning, Q.H. Yang, et al, Structural and thermal stabilities of layered $\mathrm{Li}\left(\mathrm{Ni}_{1 / 3} \mathrm{Co}_{1 / 3} \mathrm{Mn}_{1 / 3}\right) \mathrm{O}_{2}$ materials in 18650 high power batteries, J. Power Sources, 196, 10322-10327 (2011)

7. S. Liu, M. Winter, M. Lewerenz, et al, Analysis of cyclic aging performance of commercial $\mathrm{Li}_{4} \mathrm{Ti}_{5} \mathrm{O}_{12}-$ based batteries at room temperature. Energy, 173, 1041-1053 (2019)

8. P. Svens, R. Eriksson, J. Hansson, et al, Analysis of aging of commercial composite metal oxide$\mathrm{Li}_{4} \mathrm{Ti}_{5} \mathrm{O}_{12}$ battery cells, J. Power Sources, 270, 131141 (2014)

9. Y. Abdul-Quadir, T. Laurila, J. Karppinen, et al, Heat generation in high power prismatic Li-ion battery cell with $\mathrm{LiMnNiCoO}_{2}$ cathode material, Int. J. Energ. Res., 38, 1424-1437 (2014)

10. K. Smith, C.Y. Wang, Power and thermal characterization of a lithium-ion battery pack for hybrid-electric vehicles, J. Power Sources, 160, 662673 (2006) 